\title{
MicroRNA-381 regulates the occurrence and immune responses of coronary atherosclerosis via cyclooxygenase-2
}

\author{
KAIYOU SONG ${ }^{1}$, LIANTING LI ${ }^{1,2}$, GUILING SUN ${ }^{1}$ and YANJIN WEI ${ }^{1}$ \\ ${ }^{1}$ Department of Cardiology, Linyi People's Hospital, Linyi, Shandong 276003; ${ }^{2}$ Department of Internal Medicine, \\ Junan Hospital of Traditional Chinese Medicine, Linyi, Shandong 276600, P.R. China
}

Received September 28, 2016; Accepted June 5, 2017

DOI: $10.3892 /$ etm.2018.5947

\begin{abstract}
The present study aimed to measure the levels of microRNA-381 (miR-381) in the plaque tissues, peripheral blood mononuclear cells (PBMCs) and serum of patients with coronary atherosclerosis. In addition, the regulatory mechanisms of miR-381 and cyclooxygenase (COX)-2 in coronary atherosclerosis were investigated. A total of 36 patients with coronary atherosclerosis who received coronary endarterectomy at Linyi People's Hospital and Junan Hospital of Traditional Chinese Medicine (Linyi, China) between January 2013 and June 2016 were enrolled into the present study, while 39 healthy subjects were included as the control group. Peripheral blood was collected form all patients and healthy subjects. Plaque tissues were resected from patients with coronary atherosclerosis and adjacent artery intimal tissues were resected as the control tissues. Using quantitative polymerase chain reaction, the levels of miR-381 and COX-2 mRNA in the plaque tissues, PBMCs and serum were determined. In addition, COX-2 protein expression in the plaque tissues and PBMCs was measured by western blotting, while enzyme-linked immunosorbent assay was utilized to examine the protein content in the serum. To identify the direct interaction between miR-381 and COX-2 mRNA, dual-luciferase reporter assay was also conducted. The levels of COX-2 mRNA and protein in the plaque tissues, PBMCs and serum of patients with coronary atherosclerosis were significantly elevated compared with those in the corresponding control groups. However, the expression of miR-381 was significantly reduced in the coronary atherosclerosis patients. Dual-luciferase reporter assay revealed that miR-381 was able to directly target the 3'-untranslated region of COX-2 mRNA to regulate the expression of COX-2. Therefore, the present study demonstrated that enhanced levels of COX-2
\end{abstract}

Correspondence to: Dr Lianting Li, Department of Cardiology, Linyi People's Hospital, 27 Jiefang Road, Linyi, Shandong 276003, P.R. China

E-mail: cww444@163.com

Key words: microRNA-381, coronary atherosclerosis, plaques, cyclooxygenase-2 expression in patients with coronary atherosclerosis are associated with the downregulation of miR-381 expression, while miR-381 may regulate the occurrence and immune responses of coronary atherosclerosis via COX-2.

\section{Introduction}

Coronary atherosclerosis is a serious vascular disease, which may lead to coronary artery stenosis that is a common cause of angina and myocardial infarction (1). Long-term myocardial ischemia and hypoxia can result in heart enlargement, decrease of compliance and myocardial contraction dysfunction, leading to chronic heart failure (2). Coronary atherosclerotic plaque, particularly the vulnerable plaque of the coronary artery with a thrombus formation tendency, is one of the most important reasons for coronary artery stenosis (3). Coronary atherosclerosis has a greater impact on the quality of life of patients, due to its long clinical treatment, easy relapse and poor prognosis. Therefore, studies on coronary atherosclerosis at the molecular level are important for the clinical treatment of coronary atherosclerosis.

MicroRNAs (miRNAs or miRs) are a class of non-encoding RNA molecules with 18-22 nucleotides in eukaryotes, which regulate the expression of their target proteins at the mRNA level (4-6). Altered expression of various miRNA molecules and a variety of proteins in coronary atherosclerosis suggests that miRNAs serve important roles in the regulation of proteins that are associated with coronary atherosclerosis $(7,8)$. Studies have demonstrated that atherosclerosis is a type of artery intimal inflammation with a series of basic features of inflammation $(9,10)$. Furthermore, cyclooxygenase (COX)-2 is a key inflammatory factor in the human body. Activation of the COX-2 signaling pathway causes and aggravates the pathogenesis of atherosclerosis (11). Atherosclerosis can be considered chronic inflammation. COX-2 is an important rate-limiting enzyme in the synthesis of prostaglandin and thromboxane from arachidonic acid and is highly expressed at inflammatory sites (12). It has been demonstrated that COX-2 is expressed in atherosclerotic lesions and is regulated by a variety of inflammatory cytokines, including IL-1, IFN- $\gamma$ and TNF- $\alpha$ (12). The metabolites of COX-2 also promote inflammation, indicating that COX-2 is involved in the pathological process of atherosclerosis occurrence and development $(13,14)$. However, the 
mechanism of COX-2 regulation on coronary atherosclerotic plaques remains unclear.

In the present study, the expression levels of COX-2 mRNA and protein in the plaque tissues, peripheral blood mononuclear cells (PBMCs) and serum of coronary atherosclerosis patients were examined. In addition, the association between miR-381 and COX-2 was also investigated.

\section{Materials and methods}

Patients. A total of 36 patients with coronary atherosclerosis who received coronary endarterectomy at Linyi People's Hospital and Junan Hospital of Traditional Chinese Medicine (Linyi, China) between January 2013 and June 2016 were enrolled into the present study, including 19 males and 17 females (age range, 38-72 years; median age, 59 years). In addition, 39 healthy subjects were enrolled into the control group, including 18 males and 21 females (age range, 39-75 years; median age, 61 years). All patients with coronary atherosclerosis were diagnosed by electrocardiography, echocardiography and coronary angiography. None of the patients had any complications or infections in the liver or kidney, or immune-associated diseases. Subjects in the control group did not suffer from coronary atherosclerosis. The age, gender, history of diabetes and history of hypertension of all patients were recorded. Gensini scoring scale (15) was used to evaluate the degree of coronary atherosclerosis. For severity scoring, stenosis with $0-<25,25-50,50-75,75-90,90-99$ and $>90 \%$ luminal diameter reduction scored 1, 2, 3, 8, 16 and 32 points, respectively. Segment multiplying factors for the left main trunk, proximal left anterior descending branch, middle left anterior descending branch, D1 diagonal branch, D2 diagonal branches, proximal left circumflex branch, distal left circumflex branch, posterior descending branch, posterior lateral branch and the proximal, middle and distal right coronary artery was $5,2.5,1.5,1,0.5,2.5,1,1,0.5$ and 1 , respectively. The Gensini score was calculated by multiplying severity scores with their respective segment multiplying factors. All procedures were approved by the Ethics Committee of Linyi People's Hospital (Linyi, China). Written informed consents were obtained from all patients or their families.

Samples. Plaque tissues and adjacent artery intimal tissues (serving as the controls) were obtained from all patients by coronary endarterectomy and stored in liquid nitrogen until further use. In addition, peripheral blood was collected from all patients and healthy subjects (control) for the separation of serum by centrifugation at a speed of $400 \times \mathrm{g}$ at $4{ }^{\circ} \mathrm{C}$ for $10 \mathrm{~min}$. In order to obtain PBMCs, a mixture of the heparin anticoagulated venous blood and equal amount of serum-free Iscove's modified Dulbecco's medium (v/v, 1:1; P12440053; Thermo Fisher Scientific, Inc., Waltham, MA, USA) was added to the lymphocyte separation medium prior to centrifugation at a speed of $400 \mathrm{x}$ g for $30 \mathrm{~min}$ at room temperature. Following centrifugation, the middle layer was aspirated and mixed with 5 volumes of Hank's solution (H1020; Beijing Solarbio Science \& Technology Co., Ltd., Beijing, China) prior to further centrifugation at a speed of $300 \mathrm{x}$ g for $10 \mathrm{~min}$ at $4^{\circ} \mathrm{C}$. Subsequent to two washes with PBS, the cells were counted and diluted to a density of $1 \times 10^{6}$ cells $/ \mathrm{ml}$. Finally, $3 \times 10^{6}$ cells were seeded onto a round culture plate with a bottom area of $9 \mathrm{~cm}^{2}$, followed by incubation at $37^{\circ} \mathrm{C}$ and $5 \% \mathrm{CO}_{2}$ for $1-2 \mathrm{~h}$. The cells that attached on the bottom (PBMCs) were collected by discarding the medium.

Reverse transcription-quantitative polymerase chain reaction $(R T$ - $q P C R)$. Serum $(1 \mathrm{ml})$ was mixed with $1 \mathrm{ml}$ TRIzol reagent (cat. no. 10606ES60; Yeasen Biotechnology Co., Ltd., Shanghai, China) for lysis. Next, total RNA was extracted using miRNeasy Serum/Plasma kit (cat. no. JL217184; Guangzhou Jianlun Biological Technology Co., Ltd., Guangzhou, China). The purity of RNA was then determined by measuring the absorbance at 260/280 nm using ultraviolet spectrophotometry (Nanodrop ND-1000; Thermo Fisher Scientific, Inc.). Subsequently, cDNA was obtained by reverse transcription using TIANScript II cDNA First Strand Synthesis kit (cat. no. KR107; Tiangen Biotech Co., Ltd., Beijing, China) from $1 \mu \mathrm{g}$ RNA and stored at $-20^{\circ} \mathrm{C}$.

SuperReal PreMix (SYBR-Green) kit (Tiangen Biotech Co., Ltd.) was used to detect the mRNA expression of COX-2, using $\beta$-actin as an internal reference. The reaction system (total volume, $20 \mu \mathrm{l}$ ) was composed of $10 \mu \mathrm{l}$ qPCR Mix, $0.5 \mu 1$ upstream primer (COX-2 mRNA, 5'-CAGCCATAC AGCAAATCCTTG-3'; $\beta$-actin, 5'-CACCAGGGCGTGATG GT-3'), $0.5 \mu 1$ downstream primer (COX-2 mRNA, 5'-CAA ATGTGATCTGGATGTCAAC-3'; $\beta$-actin, 5'-CTCAAACAT GATCTGGGTCAT-3'), $1 \mu \mathrm{l}$ cDNA and $8 \mu 1$ dd $_{2} \mathrm{O}$. qPCR was conducted under the following condition: Initial denaturation at $95^{\circ} \mathrm{C}$ for $30 \mathrm{sec}$; 40 cycles of denaturation at $95^{\circ} \mathrm{C}$ for $5 \mathrm{sec}$ and annealing at $60^{\circ} \mathrm{C}$ for $34 \mathrm{sec}$ (iQ5 Reverse transcription PCR Detection System; Bio-Rad Laboratories, Inc., Hercules, CA, USA). The $2^{-\Delta \Delta \mathrm{Cq}}$ method (16) was used to calculate the relative expression of COX- 2 mRNA against $\beta$-actin. Each sample was tested in triplicate.

The expression of miR-381 was determined by miRcute miRNA qPCR detection kit (cat. no. FP401; Tiangen Biotech Co., Ltd.), using U6 as an internal reference. The reaction system (total volume, $25 \mu \mathrm{l}$ ) contained $12.5 \mu \mathrm{l}$ SYBR Premix Ex Taq, $1 \mu 1$ upstream primer (miR-381, 5'-ACACTCCAG CTGGGTATACAAGGGCAAGCT-3'; U6, 5'-CTCGCTTCG GCAGCACA-3'), $1 \mu \mathrm{l}$ downstream primer (miR-381, 5'-TGG TGTCGTGGAGTCG-3'; U6, 5'-AACGCTTCACGAATT

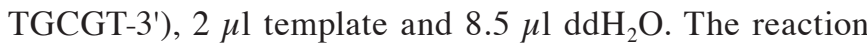
protocol was as follows: Initial denaturation at $95^{\circ} \mathrm{C}$ for $5 \mathrm{~min}$; 40 cycles of denaturation at $95^{\circ} \mathrm{C}$ for $15 \mathrm{sec}$, annealing at $60^{\circ} \mathrm{C}$ for $30 \mathrm{sec}$ and elongation at $72^{\circ} \mathrm{C}$ for $30 \mathrm{sec}$ (iQ5; Bio-Rad Laboratories, Inc.). The $2^{-\Delta \Delta \mathrm{Cq}}$ method was used to calculate the relative expression of miR-381 against U6. Each sample was tested in triplicate.

Western blotting. Tissues and cells were collected and mixed with $100 \mu \mathrm{l}$ precooled radioimmunoprecipitation assay lysis buffer (P0013B; Beyotime Institute of Biotechnology, Shanghai, China) containing $1 \mathrm{mM}$ phenylmethylsulfonyl fluoride for lysis for $15 \mathrm{~min}$ at $4^{\circ} \mathrm{C}$. Next, the mixture was centrifuged at $12,000 \mathrm{x} \mathrm{g}$ at $4^{\circ} \mathrm{C}$ for $5 \mathrm{~min}$. The supernatant was used to determine the protein concentration with a bicinchoninic acid protein concentration determination kit (cat. no. RTP7102; Real-Times Biotechnology Co., Ltd., Beijing, China). Protein samples were then mixed with sodium dodecyl 
sulfate (SDS) loading buffer prior to denaturation in a boiling water bath for $5 \mathrm{~min}$. Subsequently, the samples $(20 \mu \mathrm{g})$ were subjected to $10 \%$ SDS-polyacrylamide gel electrophoresis. The resolved proteins were transferred to polyvinylidene difluoride membranes on ice $(100 \mathrm{~V} ; 2 \mathrm{~h})$ and blocked with $5 \%$ skimmed milk at room temperature for $1 \mathrm{~h}$. The membranes were then incubated with rabbit anti-human COX-2 polyclonal primary antibody (1:1,000; cat. no. ab15191; Abcam, Cambridge, UK) and rabbit anti-human $\beta$-actin primary antibody (1:5,000; cat. no. ab129348; Abcam) at $4^{\circ} \mathrm{C}$ overnight. Following washing three times for $5 \mathrm{~min}$ each, with phosphate-buffered saline with Tween-20 (TBST), the membranes were incubated with polyclonal goat anti-rabbit horseradish peroxidase-conjugated secondary antibody (1:3,000; cat. no. ab6721; Abcam) for $1 \mathrm{~h}$ at room temperature, followed by washing with TBST for three times for $15 \mathrm{~min}$ each. Subsequently, the membrane was developed with an enhanced chemiluminescence detection kit (cat. no. ab65623; Abcam) for imaging. Image lab version 3.0 software (Bio-Rad Laboratories, Inc.) was used to acquire and analyze imaging signals. The relative expression of COX-2 protein was calculated against that of $\beta$-actin.

Enzyme-linked immunosorbent assay (ELISA). Blood samples were centrifuged at a speed of $1,000 \mathrm{xg}$ at $4^{\circ} \mathrm{C}$ for $10 \mathrm{~min}$ and serum was tested using a COX-2 ELISA kit (cat. no. KB1567; Shanghai Kemin Biotech Co., Ltd., Shanghai, China). Standards (50 $\mu \mathrm{l}$; provided by the kit), serum samples (10 $\mu \mathrm{l}$ sample liquid and $40 \mu \mathrm{l}$ diluent) and blanks were added into the predefined wells of a 96-well microplate. In the standard and serum wells, horseradish peroxidase-labelled conjugates $(100 \mu \mathrm{l})$ were added prior to sealing the plates for incubation at $37^{\circ} \mathrm{C}$ for $1 \mathrm{~h}$. Subsequent to five washes using wash liquid (part of the kit), substrates A $(50 \mu \mathrm{l})$ and B $(50 \mu \mathrm{l})$ were added into each well. After incubation at $37^{\circ} \mathrm{C}$ for $15 \mathrm{~min}$, stop solution $(50 \mu \mathrm{l})$ was added into each well and the absorbance was measured at $450 \mathrm{~nm}$ within $15 \mathrm{~min}$.

Dual-luciferase reporter assay. Bioinformatics prediction is a powerful tool used to study the functions of miRNAs. In order to understand the regulatory mechanism of COX-2 in coronary atherosclerosis, the online software miRanda (www.microma. org/rnicroma/home.do), TargetScan (www.targetscan.org), PITA (genie.weizmann.ac.il/pubs/mir07/mir07_data.html), RNAhybrid (bibiserv.techfak.uni-bielefeld.de/rnahybrid) and PicTar (pictar.mdc-berlin.de) were used to predict miRNA molecules that may regulate $\mathrm{COX}-2$. The analysis identified that miR-381 was able to potentially regulate COX-2.

The cells were divided into negative control (NC), wild-type (WT) and mutant groups. According to the bioinformatics results, WT and mutant seed regions of miR-381 in the 3'-untranslated region (UTR) of the COX-2 gene were chemically synthesized in vitro, added with SpeI and HindIII restriction sites for the insertion of plasmids and then cloned into pMIR-REPORT luciferase reporter plasmids (Ambion; Thermo Fisher Scientific, Inc.). Plasmids $(0.8 \mu \mathrm{g})$ with WT or mutant 3'-UTR DNA sequences were co-transfected with agomiR-381 (100 nM; Sangon Biotech Co., Ltd., Shanghai, China) into 293T cells [ATCC, Manassas, VA, USA; cultured in 24-well plates containing Dulbecco's modified Eagle's medium (Thermo Fisher Scientific, Inc.) and 10\% fetal bovine serum
(Sangon Biotech Co. Ltd.) at $37^{\circ} \mathrm{C}$ and $5 \% \mathrm{CO}_{2}$ ]. Following cultivation for $24 \mathrm{~h}$, the cells were lysed using a dual-luciferase reporter assay kit (Promega Corporation, Madison, WI, USA) according to the manufacturer's protocol and fluorescence intensity was measured using a GloMax 20/20 luminometer (Promega Corporation). Using Renilla fluorescence activity as an internal reference, the fluorescence values of each group of cells were measured.

Statistical analysis. The results were analyzed using SPSS version 18.0 statistical software (SPSS, Inc., Chicago, IL, USA). The data are expressed as the mean \pm standard deviation. Data were tested for normality and multigroup measurement data were analyzed using one-way analysis of variance. In case of homogeneity of variance, the least significant difference and Student-Newman-Keuls methods were used, whereas in case of heterogeneity of variance, Tamhane's T2 or Dunnett's T3 method was used. $\mathrm{P}<0.05$ indicated statistically significant differences.

\section{Results}

COX-2 mRNA expression is upregulated in the plaque tissues, PBMCs and serum of patients with coronary atherosclerosis. To measure the expression of COX-2 mRNA, RT-qPCR was employed. The data demonstrated that the levels of COX-2 mRNA in the plaques, PBMCs and serum of patients with coronary atherosclerosis were significantly higher when compared with those in normal adjacent tissues or healthy subjects $(\mathrm{P}<0.05$; Fig. 1). These results suggested that upregulation of COX-2 mRNA in plaque tissues, PBMCs and serum was associated with the occurrence of coronary atherosclerosis.

Increased expression of $C O X-2$ protein in plaque tissues and PBMCs suggests its regulatory role in coronary atherosclerosis. To determine COX-2 protein expression in plaque tissues and PBMCs, western blotting was used. The data revealed that COX-2 protein levels in plaque tissues and PBMCs from patients with coronary atherosclerosis were significantly elevated compared with those in the corresponding control groups $(\mathrm{P}<0.05$; Fig. 2). The results indicated that increased expression of COX-2 protein in the plaque tissues and PBMCs may serve a regulatory role in coronary atherosclerosis.

Secretion of COX-2 protein into the blood by PBMCs is promoted in coronary atherosclerosis. To examine the contents of COX-2 protein in the serum, ELISA was conducted. The data indicated that the serum level of COX-2 protein in patients with coronary atherosclerosis was significantly higher in comparison with that in healthy subjects $(\mathrm{P}<0.05$; Fig. 3). These findings suggested that the secretion of COX-2 protein into the blood by PBMCs was promoted in coronary atherosclerosis.

miR-381 may serve a regulatory role in the pathogenesis of coronary atherosclerosis. To study the expression of miR-381, RT-qPCR was performed. The results revealed that the levels of miR-381 in plaque tissues, PBMCs and serum obtained from patients with coronary atherosclerosis were significantly reduced as compared with the corresponding 
A

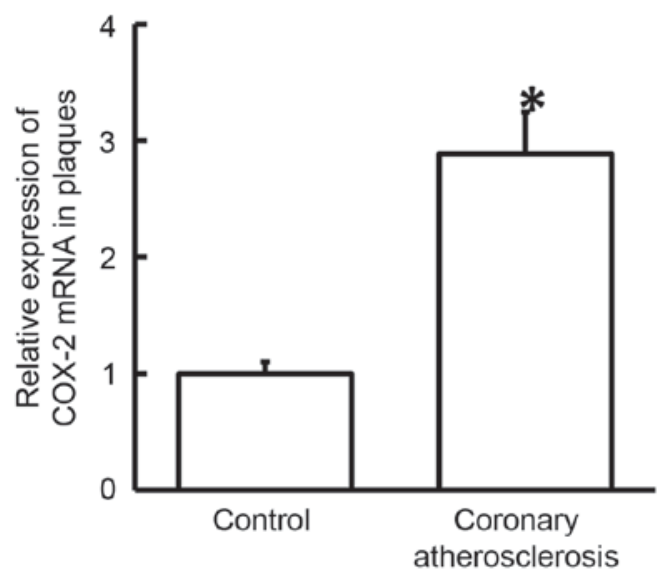

C

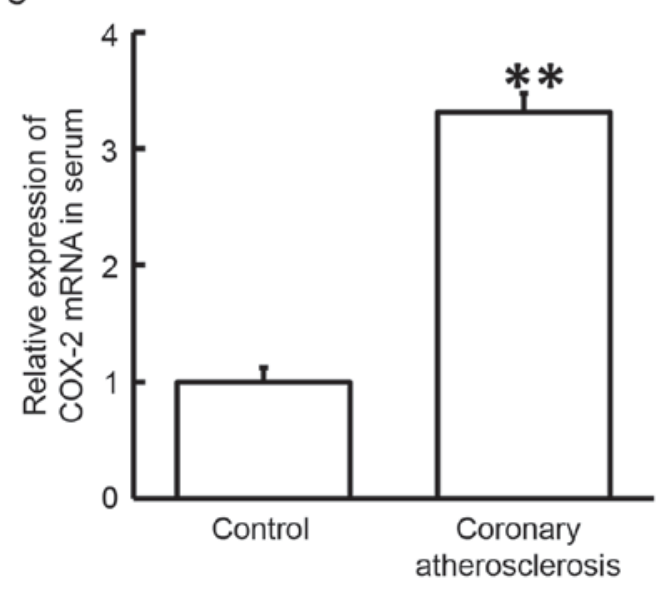

B

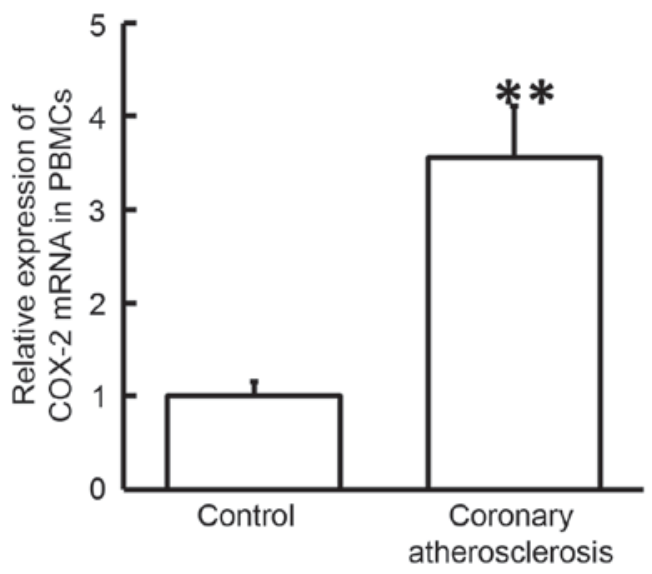

Figure 1. Expression of COX-2 mRNA in the (A) plaque tissues, (B) PBMCs and (C) serum of healthy subjects and patients with coronary atherosclerosis. Reverse transcription-quantitative polymerase chain reaction was used to measure the expression of COX-2 mRNA. ${ }^{*} \mathrm{P}<0.05$ and ${ }^{* *} \mathrm{P}<0.01$ vs. corresponding control group (adjacent tissues from patients or blood samples from healthy subjects). COX-2, cyclooxygenase-2; PBMCs, peripheral blood mononuclear cells.

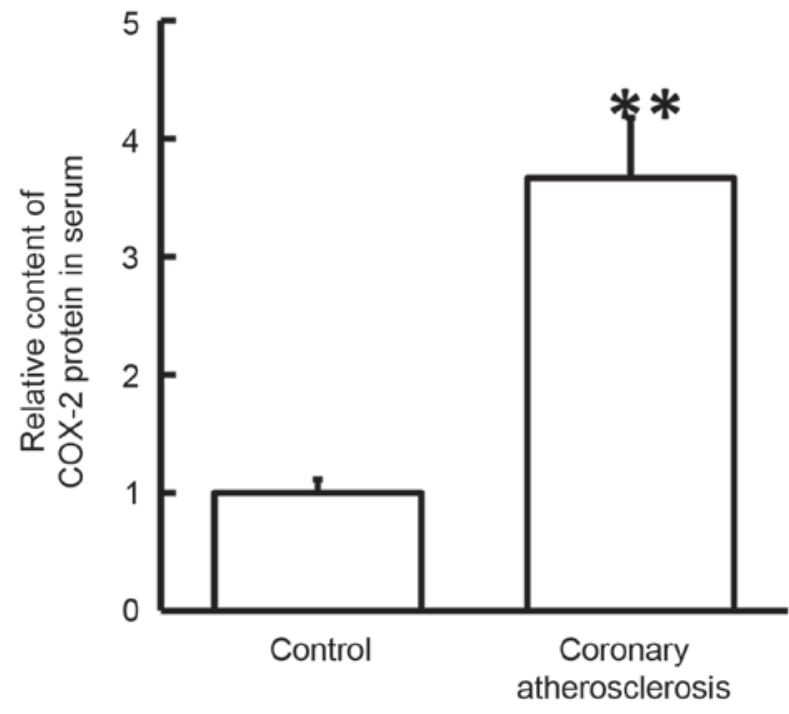

Figure 2. Serum content of COX-2 protein in healthy subjects and patients with coronary atherosclerosis, determined by enzyme-linked immunosorbent assay. ${ }^{* *} \mathrm{P}<0.01$ vs. control group. COX-2, cyclooxygenase-2.

control groups $(\mathrm{P}<0.05$; Fig. 4). These results indicate that miR-381 may serve a regulatory role in the pathogenesis of coronary atherosclerosis.
miR-381 regulates the expression of COX-2 by directly binding with the 3'-UTR of COX-2. Analyses performed using miRanda, TargetScan, PITA, RNAhybrid and PicTar demonstrated that miR-381 may target COX-2 (data not shown). To test whether miR-381 directly targets COX-2, dual-luciferase reporter assay was performed. The data demonstrated that transfection with agomiR-381 and pMIR-REPORT in the WT group resulted in a significantly reduced fluorescence intensity compared with the negative control $(\mathrm{P}<0.05)$. By contrast, fluorescence intensity in the mutant group was not significantly different from that in the negative control $(\mathrm{P}>0.05$; Fig. 5). These observations suggest that miR-381 regulated the expression of COX-2 by directly binding with the 3'-UTR of COX-2.

\section{Discussion}

During the occurrence and development of chronic inflammation, COX-2 serves an important role. COX-2 is induced by external stimuli (physical, chemical or biological factors) and participates in inflammatory reactions by catalyzing the synthesis of prostaglandins (17). Due to the importance of COX-2 in inflammation, high expression of COX-2 in tissues has become a significant marker of inflammation (18). In addition to antipyretic and analgesic effects, COX-2 inhibitors 
A

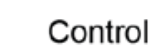

Coronary

$\operatorname{cox}-2$

$\beta$-actin
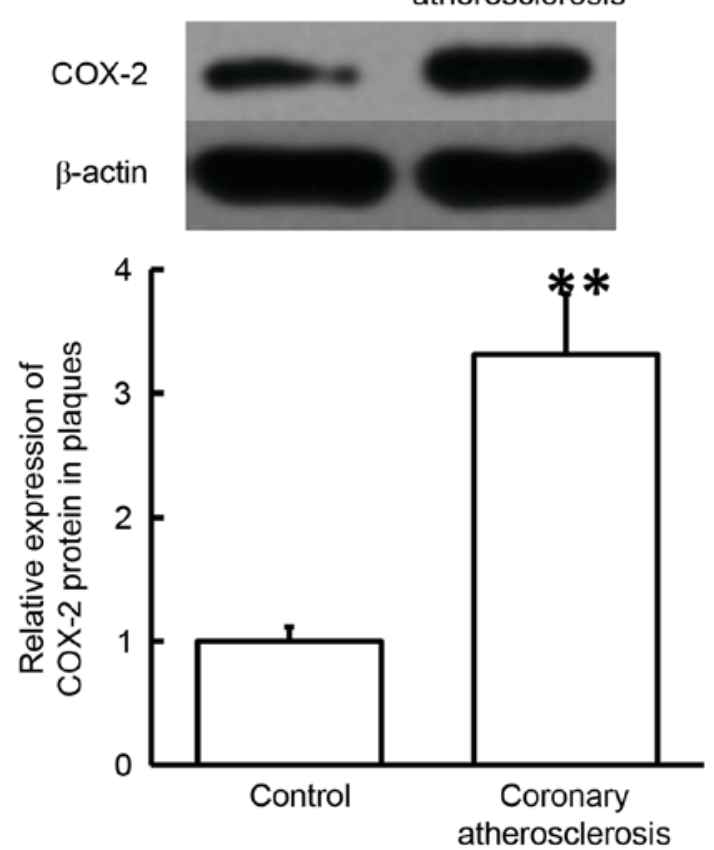

B Control Coronary
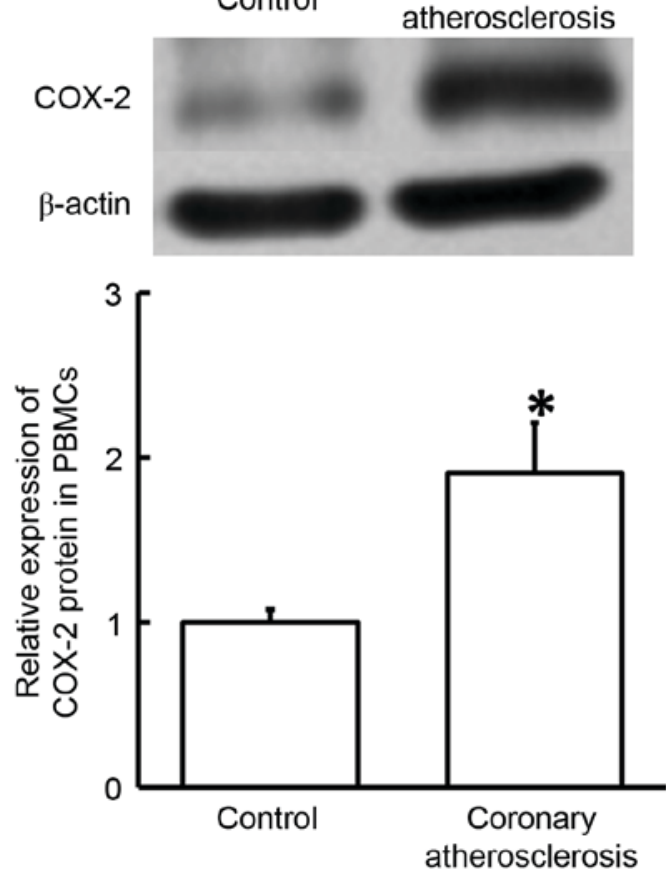

Figure 3. Expression of COX-2 protein in the (A) plaque tissues and (B) PBMCs obtained from patients with coronary atherosclerosis. Western blotting was employed to measure the protein expression. ${ }^{*} \mathrm{P}<0.05$ and ${ }^{* *} \mathrm{P}<0.01$ vs. control group. COX-2, cyclooxygenase-2; PBMCs, peripheral blood mononuclear cells.

A

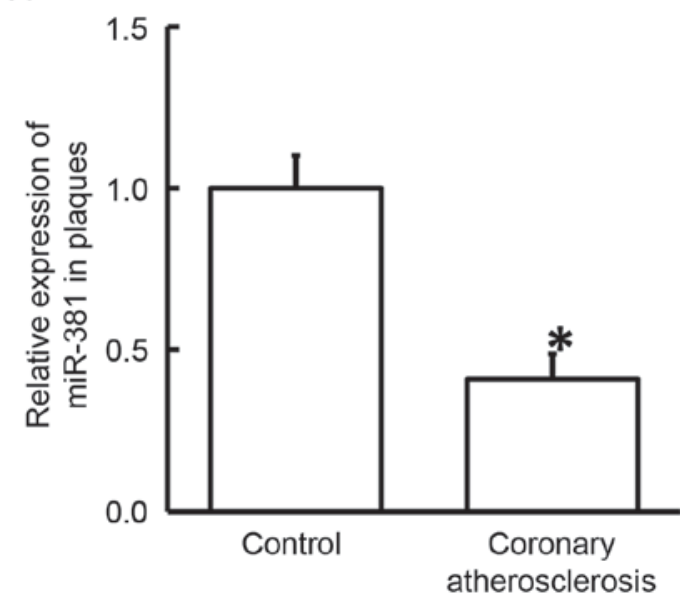

C

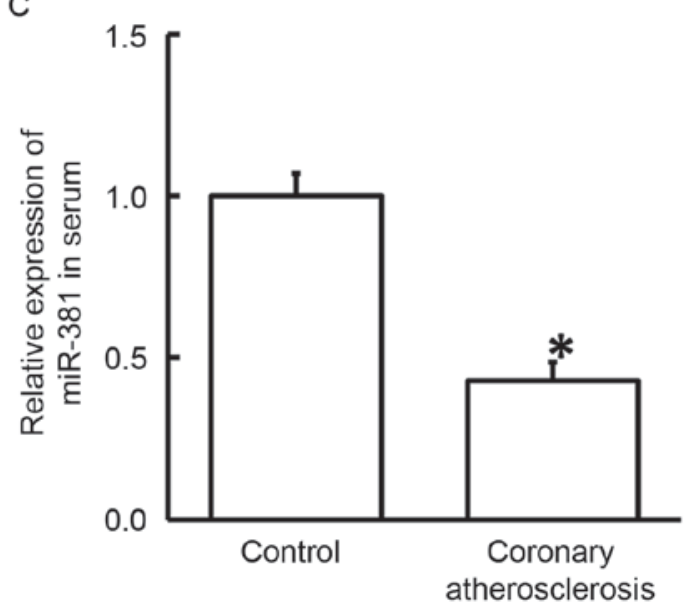

B

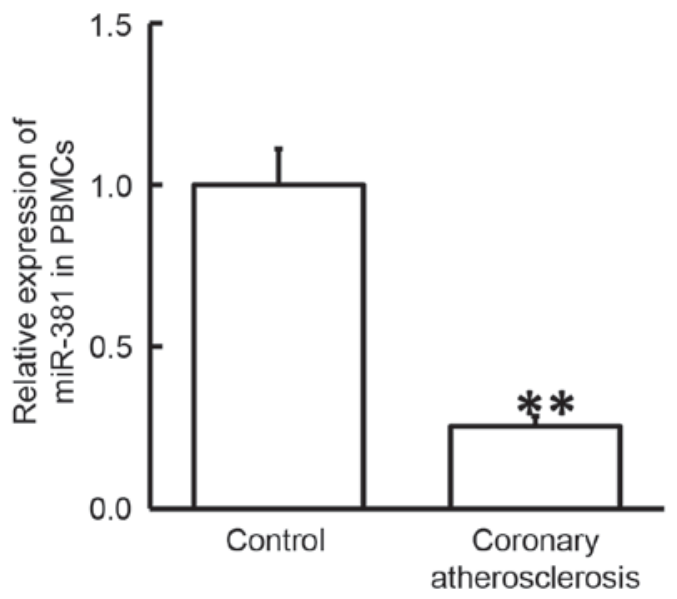

Figure 4. Expression of miR-381 in the (A) plaque tissues, (B) PBMCs and (C) serum of healthy subjects and patients with coronary atherosclerosis. Reverse transcription-quantitative polymerase chain reaction was used to determine the expression of $\mathrm{miR}-381$. ${ }^{*} \mathrm{P}<0.05$ and ${ }^{* *} \mathrm{P}<0.01$ vs. the control group. $\mathrm{COX}-2$, cyclooxygenase-2; PBMCs, peripheral blood mononuclear cells; miR, microRNA. 


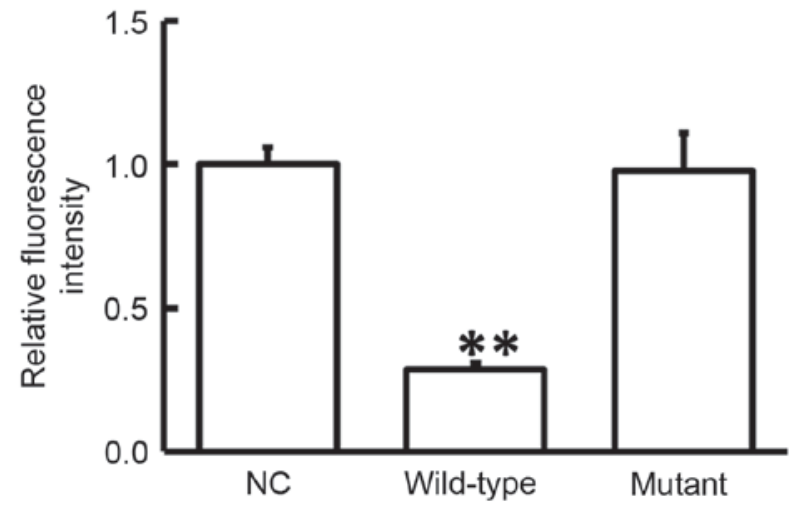

Figure 5. Fluorescence intensity of $293 \mathrm{~T}$ cells transfected with wild-type or mutant 3'-untranslated region DNA sequences of COX-2 and agomiR-381. Dual-luciferase reporter assay was used to evaluate the interaction between miR-381 and COX-2. ${ }^{* *} \mathrm{P}<0.01$ vs. NC group. COX-2, cyclooxygenase-2; NC, negative controls.

can also serve an auxiliary role in antitumor therapy (19). In previous studies, the association between COX-2 and atherosclerosis has been examined. It has been reported that the genetic variation of COX-2 is associated with cardiovascular diseases (20). Another study observed that endotoxin-treated porcine coronary artery contraction induced by bradykinin was achieved via the COX-2 pathway (21). Furthermore, COX-2 is considered to exert certain therapeutic effects in the process of atherosclerosis by selectively inhibiting celecoxib (22). Consistently, the results of the present study revealed that the expression of COX-2 was elevated in the plaque tissues, PBMCs and serum of coronary atherosclerosis patients, suggesting that the onset of this disease is similar to that of vascular intimal inflammation, in which PBMCs and lymphocytes are activated. These cells secrete a great amount of COX-2 to initiate abundant antigen immune responses.

Previous studies have suggested that miRNA molecules are important in the occurrence and development of various diseases $(23,24)$ and may slice the mRNA of COX-2 and inhibit its translation (25). It has been reported that miR-381 is downregulated in colon cancer tissues, leading to upregulated liver receptor homolog-1, as well as the proliferation and invasion of colon cancer cells (26). In addition, miR-381, which exists in the negative feedback loop of p53/pituitary tumor-transforming 1 , inhibits the growth of hypophysoma (27). miR-381 is also closely associated with multidrug resistance protein 1 gene and serves important roles in multidrug resistance (28). Along with miR-424, miR-381 can target WEE1 gene and inhibit the activity of cyclin-dependent kinase 2 in renal carcinoma cells (29). Furthermore, miR-381 is also closely associated with lung adenocarcinoma (30). These previous studies suggested that miR-381 expression may be closely correlated with various human diseases.

According to bioinformatics prediction conducted in the present study, miR-381 was predicted to be a potential upstream regulator of COX-2. The data of the present study suggested that miR-381 expression was downregulated and COX-2 was upregulated in the plaques and PBMCs of coronary atherosclerosis patients. This suggests that the body may decrease miR-381 levels to negatively regulate its slicing effect on $\mathrm{COX}-2$, while enhanced expression of $\mathrm{COX}-2$ then produces immune responses. Similar results were observed in the serum of patients, indicating that increased COX-2 in PBMCs can also be released into the blood. Therefore, the levels of miR-381 and COX-2 in the serum may reflect the degrees of inflammation responses and tissue injuries in coronary atherosclerosis. Notably, the current study data of dual-luciferase reporter assay revealed that miR-381 directly binds with the 3'-UTR of COX-2 mRNA and regulates the expression of COX-2.

In conclusion, the present study demonstrated that enhanced levels of COX-2 expression in patients with coronary atherosclerosis were associated with the downregulation of miR-381 expression. Thus, miR-381 may regulate the occurrence and immune responses of coronary atherosclerosis via COX-2.

\section{Acknowledgements}

The authors would like to thank the doctors and nurses at the Linyi People's Hospital (Linyi, China) who assisted in the plasma specimen and clinical data collection.

\section{Funding}

No funding was received.

\section{Availability of data and materials}

The analyzed data sets generated during the present study are available from the corresponding author on reasonable request.

\section{Authors' contributions}

KS and LL conceived and designed the study. LL and GS performed the experiments and collected the data. KS and YW wrote the paper and interpretated the data. KS and LL reviewed and edited the manuscript. All authors read and approved the manuscript.

\section{Ethics approval and consent to participate}

All procedures were approved by the Ethics Committee of Linyi People's Hospital (Linyi, China). Written informed consents were obtained from all patients or their families.

\section{Consent for publication}

Not applicable.

\section{Competing interests}

The authors declare that they have no competing interests.

\section{References}

1. Alexy T, Pais E, Wenby RB, Mack WJ, Hodis HN, Kono N, Wang J, Baskurt OK, Fisher TC and Meiselman HJ: Abnormal blood rheology and chronic low grade inflammation: Possible risk factors for accelerated atherosclerosis and coronary artery disease in Lewis negative subjects. Atherosclerosis 239: 248-251, 2015.

2. Kucharska-Newton AM, Heiss G, Ni H, Stearns SC, Puccinelli-Ortega N, Wruck LM and Chambless L: Identification of heart failure events in medicare claims: The atherosclerosis risk in communities (ARIC) study. J Card Fail 22: 48-55, 2016. 
3. Naghavi M, Libby P, Falk E, Casscells SW, Litovsky S, Rumberger J, Badimon JJ, Stefanadis C, Moreno P, Pasterkamp G, et al: From vulnerable plaque to vulnerable patient: A call for new definitions and risk assessment strategies: Part I. Circulation 108: 1664-1672, 2003.

4. Jiang XI, Luo Y, Zhao S, Chen Q, Jiang C, Dai Y, Chen Y and Cao Z: Clinical significance and expression of microRNA in diabetic patients with erectile dysfunction. Exp Ther Med 10 213-218, 2015.

5. Jia W, Wu Y, Zhang Q, Gao GE, Zhang C and Xiang Y: Expression profile of circulating microRNAs as a promising fingerprint for cervical cancer diagnosis and monitoring. Mol Clin Oncol 3: $851-858,2015$

6. Graziano A, Lo Monte G, Piva I, Caserta D, Karner M, Engl B and Marci R: Diagnostic findings in adenomyosis: A pictorial review on the major concerns. Eur Rev Med Pharmacol Sci 19: $1146-1154,2015$

7. Tang Y, Zhang YC, Chen Y, Xiang Y, Shen CX and Li YG: The role of miR-19b in the inhibition of endothelial cell apoptosis and its relationship with coronary artery disease. Sci Rep 5: 15132, 2015.

8. Ouimet M, Ediriweera HN, Gundra UM, Sheedy FJ, Ramkhelawon B, Hutchison SB, Rinehold K, van Solingen C, Fullerton MD and Cecchini K, et al: MicroRNA-33-dependent regulation of macrophage metabolism directs immune cell polarization in atherosclerosis. J Clin Invest 125: 4334-4348, 2015

9. Ross R: The pathogenesis of atherosclerosis-an update. N Engl J Med 314: 488-500, 1986.

10. Ross R: Atherosclerosis-an inflammatory disease. N Engl J Med 340: 115-126, 1999.

11. Oguz N, Kirca M, Cetin A and Yesilkaya A: Effect of uric acid on inflammatory COX-2 and ROS pathways in vascular smooth muscle cells. J Recept Signal Transduct Res 37: 500-505, 2017.

12. Rumzhum NN and Ammit AJ: Cyclooxygenase 2: Its regulation, role and impact in airway inflammation. Clin Exp Allergy 46: 397-410, 2016.

13. Schonbeck U, Sukhova GK, Graber P, Coulter S and Libby P: Augmented expression of cyclooxygenase-2 in human atherosclerotic lesions. Am J Pathol 155: 1281-1291, 1999.

14. Baker CS, Hall RJ, Evans TJ, Pomerance A, Maclouf J, Creminon C, Yacoub MH and Polak JM: Cyclooxygenase-2 is widely expressed in atherosclerotic lesions affecting native and transplanted human coronary arteries and colocalizes with inducible nitric oxide synthase and nitrotyrosine particularly in macrophages. Arterioscler Thromb Vasc Biol 19: 646-655, 1999.

15. Dursunoglu N, Dursunoglu D, Yildiz AI, Uludag B, Alaçam ZN and Sarıçopur A: Severity of coronary atherosclerosis in patients with COPD. Clin Respir J 11: 751-756, 2017.

16. Livak KJ and Schmittgen TD: Analysis of relative gene expression data using real-time quantitative PCR and the 2(-Delta Delta C(T)) method. Methods 25: 402-408, 2001.

17. Alhouayek M and Muccioli GG: COX-2-derived endocannabinoid metabolites as novel inflammatory mediators. Trends Pharmacol Sci 35: 284-292, 2014.
18. Motino O, Agra N, Brea Contreras R, Domínguez-Moreno M, García-Monzón C, Vargas-Castrillón J, Carnovale CE, Boscá L, Casado M, Mayoral R, et al: Cyclooxygenase-2 expression in hepatocytes attenuates non-alcoholic steatohepatitis and liver fibrosis in mice. Biochim Biophys Acta 1862: 1710-1723, 2016.

19. Kim HJ, Yim GW, Nam EJ and Kim YT: Synergistic effect of COX-2 inhibitor on paclitaxel-induced apoptosis in the human ovarian cancer cell line OVCAR-3. Cancer Res Treat 46: 81-92, 2014.

20. Ross S, Eikelboom J, Anand SS, Eriksson N, Gerstein HC, Mehta S, Connolly SJ, Rose L, Ridker PM, Wallentin L, et al: Association of cyclooxygenase-2 genetic variant with cardiovascular disease. Eur Heart J 35: 2242-2248a, 2014.

21. More AS, Kim HM, Zhao R, Khang G, Hildebrandt T, Bernlöhr C, Doods H, Lee D, Lee SH, Vanhoutte PM and Wu D: COX-2 mediated induction of endothelium-independent contraction to bradykinin in endotoxin-treated porcine coronary artery. J Cardiovasc Pharmacol 64: 209-217, 2014.

22. Papageorgiou N, Zacharia E, Briasoulis A, Charakida M and Tousoulis D: Celecoxib for the treatment of atherosclerosis. Expert Opin Investig Drugs 25: 619-633, 2016.

23. Lewis BP, Burge CB and Bartel DP: Conserved seed pairing, often flanked by adenosines, indicates that thousands of human genes are microRNA targets. Cell 120: 15-20, 2005.

24. Chen K and Rajewsky N: The evolution of gene regulation by transcription factors and microRNAs. Nat Rev Genet 8: 93-103, 2007.

25. Liu D, Wang D, Xu Z, Gao J, Liu M, Liu Y, Jiang M and Zheng D: Dysregulated expression of miR-101b and miR-26b lead to age-associated increase in LPS-induced COX-2 expression in murine macrophage. Age (Dordr) 37: 97, 2015.

26. Liang Y, Zhao Q, Fan L, Zhang Z, Tan B, Liu Y and Li Y: Down-regulation of MicroRNA-381 promotes cell proliferation and invasion in colon cancer through up-regulation of LRH-1. Biomed Pharmacother 75: 137-141, 2015.

27. Liang HQ, Wang RJ, Diao CF, Li JW, Su JL and Zhang S: The PTTG1-targeting miRNAs miR-329, miR-300, miR-381 and miR-655 inhibit pituitary tumor cell tumorigenesis and are involved in a p53/PTTG1 regulation feedback loop. Oncotarget 6 : 29413-29427, 2015.

28. Xu Y, Ohms SJ, Li Z, Wang Q, Gong G, Hu Y, Mao Z, Shannon MF and Fan JY: Changes in the expression of miR-381 and miR-495 are inversely associated with the expression of the MDR1 gene and development of multi-drug resistance. PLoS One 8: e82062, 2013.

29. Chen B, Duan L, Yin G, Tan J and Jiang X: Simultaneously expressed miR-424 and miR-381 synergistically suppress the proliferation and survival of renal cancer cells-Cdc2 activity is up-regulated by targeting WEE1. Clinics (Sao Paulo) 68: 825-833, 2013

30. Rothschild SI, Tschan MP, Jaggi R, Fey MF, Gugger M and Gautschi O: MicroRNA-381 represses ID1 and is deregulated in lung adenocarcinoma. J Thorac Oncol 7: 1069-1077, 2012. 